\title{
Search for the Higgs particle in models beyond the MSSM
}

\author{
James Olsen ${ }^{* \dagger}$ \\ Princeton University \\ E-mail: jolseneprinceton.edu
}

\begin{abstract}
The precise nature of the boson recently discovered at the Large Hadron Collider remains open. In addition to measuring its properties to determine whether it is the quantum of the field that breaks electroweak symmetry and gives mass to the fundamental particles, the search for other Higgs particles predicted by models beyond the standard model (SM), and also models beyond the minimal supersymmetric standard model (MSSM), has intensified. In this report, results are presented from three searches for Higgs-like particles predicted in models beyond the MSSM: a standard model with four generations (SM4), dimuon decays of a Higgs particle in the Next-toMSSM (NMSSM) framework, and doubly charged Higgs bosons predicted by the minimal type II seesaw model. The analysed samples include up to $5 \mathrm{fb}^{-1}$ of proton-proton collision data recorded by the CMS detector at a centre-of-mass energy of $7 \mathrm{TeV}$.
\end{abstract}

36th International Conference on High Energy Physics

4-11 July 2012

Melbourne, Australia

\footnotetext{
* Speaker.

†n behalf of the CMS Collaboration.
} 
Recently, the discovery of a new boson at the LHC has been reported by the CMS [1] and ATLAS [2] collaborations. The evidence of a Higgs-like sector to the SM is now compelling, but a definitive conclusion on the nature of this particle remains open. Whether it is the Higgs boson predicted by the SM or not, searches for other Higgs-like particles now take on increased importance. This report summarizes searches for three Higgs particles predicted in models beyond the SM, and beyond the MSSM: a standard model with four generations (SM4), dimuon decays of a Higgs particle in the next-to MSSM (NMSSM) framework, and doubly charged Higgs bosons predicted by the minimal type II seesaw Model. The data used consist of up to $5 \mathrm{fb}^{-1}$ of protonproton collisions recorded by the CMS detector [3] at a centre-of-mass energy of $7 \mathrm{TeV}$.

\section{Search for SM4 Higgs Boson}

Searches for the SM Higgs boson can be reinterpreted in the context of an extension with four generations [4], where the only differences are the assumed cross sections and branching fractions. For example, in this context the gluon fusion production process is enhanced by up to an order of magnitude due to the presence of fourth generation particles $\left(\mathrm{t}^{\prime}\right.$ and $\left.\mathrm{b}^{\prime}\right)$ replacing the top quark mediating the $\mathrm{gg} \rightarrow \mathrm{H}$ loop diagram. Prior to the work reported here, direct experimental searches for the SM4 Higgs boson excluded the mass range 131-207 GeV [5, 6].

The SM4 benchmark parameter set recommended by the LHC Higgs Cross Section Working Group [7] is used: $m_{\ell_{4}}=m_{v_{4}}=m_{d_{4}}=600 \mathrm{GeV}$ and $m_{u_{4}}-m_{d_{4}}=\left(50+10 \cdot \ln \left(m_{\mathrm{H}} / 115\right)\right) \mathrm{GeV}$. Here, $m_{\ell_{4}\left(v_{4}\right)}$ and $m_{u_{4}\left(d_{4}\right)}$ are the fourth generation charged (neutral) lepton and up (down) quark, respectively. In this model, the effective squared couplings of a SM4 Higgs boson to $\mathrm{W}$ and Z bosons become about three times smaller, while that of photons is suppressed by about two orders of magnitude due to a cancellation of SM and SM4 diagrams. The squared couplings to fermions are increased by approximately $60 \%$. Due to the modified couplings, the gluon-fusion production cross section is enhanced by up to an order of magnitude relative to those of the vector-boson fusion and vector-boson associated production mechanisms. The latter are therefore reset to zero in the analysis. At the time of this conference, the only results available from CMS on the decay to bottom quarks used the $\mathrm{VH}$ production process, so this decay channel is absent in the combination. Therefore, the final combination includes the previously published $\gamma \gamma$ [8], WW [9], ZZ [10], and $\tau \tau[11]$ searches only.

No significant signal is observed, and 95\% confidence level (CL) upper limits are computed as a function of the SM4 Higgs boson mass. Figure 1 shows results for the upper limit on the signal strength parameter $\mu$, defined as the ratio of the observed (or expected) cross section relative to that expected for SM4 Higgs boson $(\mu=\sigma / \sigma(\mathrm{SM} 4))$. Results are shown for the full search region $(110-600 \mathrm{GeV})$, and a reduced range showing the low-mass region in more detail. The entire search region was expected to be excluded in the case that there is no SM4 Higgs boson, while the observed exclusion is in the range $123<m_{\mathrm{H}}<600 \mathrm{GeV}$. These results are documented in Ref. [12].

\section{Search for a Light Pseudoscalar Higgs Boson Decaying to Muons}

In the NMSSM (see Ref. [13] for a review) a complex singlet superfield is introduced, ex- 

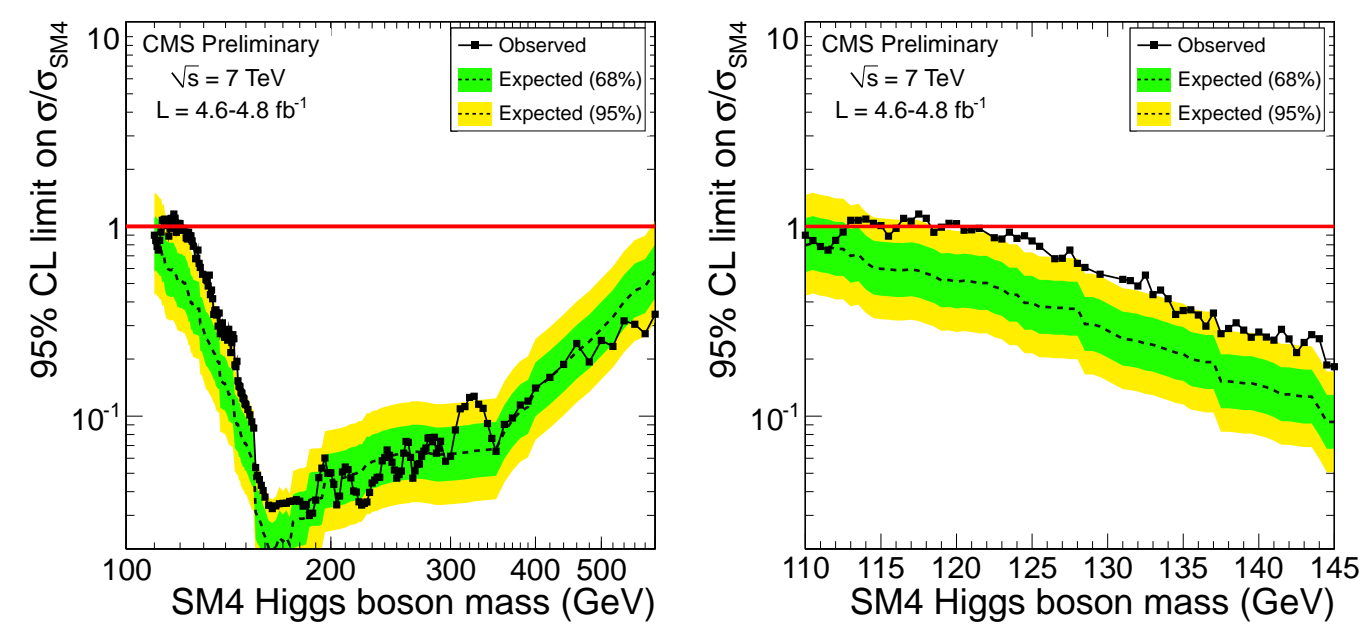

Figure 1: Results of the search for a SM4 Higgs boson, showing the expected (dashed) and observed (solid) 95\% confidence level upper limits on the signal strength parameter $\mu$ (see text) as a function of the mass. The left (right) plot shows results over the full (reduced) mass range.

tending the family of Higgs bosons to seven: three CP-even scalars $\left(h_{1}, h_{2}, h_{3}\right)$, two CP-odd scalars $\left(a_{1}, a_{2}\right)$, and two charged scalars $\left(\mathrm{H}^{+}, \mathrm{H}^{-}\right)$. The $a_{1}$ is a superposition of the MSSM doublet pseudoscalar $\left(a_{\mathrm{MSSM}}\right)$ and the additional singlet pseudoscalar of the $\operatorname{NMSSM}\left(a_{\mathrm{S}}\right): a_{1}=$ $\cos \theta_{A} \cdot a_{\mathrm{MSSM}}+\sin \theta_{A} \cdot a_{\mathrm{S}}$, where $\theta_{A}$ is the mixing angle between the two pseudoscalars. In the context of the NMSSM, the $a_{1}$ can have a small mass and be nearly pure $a_{\mathrm{S}}\left(\cos \theta_{A}<<1\right)$, while still generating a significant coupling to muons, taus, and bottom quarks for large $\tan \beta$, the ratio of the vacuum expectation values for the two Higgs doublets [14].

The analysis presented here searches for dimuon decays of a light pseudoscalar Higgs boson generically referred to as $a$ and having a mass near the Upsilon family of resonances. The data comprise $1.3 \mathrm{fb}^{-1}$ collected with a dedicated trigger and the search focuses on two regions: "mass range 1" extending from 5.5 to $8.8 \mathrm{GeV}$, and "mass range 2" extending from 11.5 to $14 \mathrm{GeV}$. The $a$ is expected to be very narrow $(\sim \mathrm{MeV})$, and thus the observed peak should be dominated by the known dimuon mass resolution of the CMS detector, which ranges from a minimum of $50 \mathrm{MeV}$ in the lower mass range to a maximum of $190 \mathrm{MeV}$ in the upper mass range.

Events were collected with a trigger requiring two opposite-sign muons with each muon having a momentum transverse to the beam satisfying $p_{\mathrm{T}}>3.5 \mathrm{GeV}$, and a distance of closest approach to the beam line compatible with a prompt decay. The dimuon system must further satisfy $p_{\mathrm{T}}>$ $6 \mathrm{GeV}$ and $5.5<m_{\mu \mu}<14 \mathrm{GeV}$. The trigger had a prescale factor of 2 applied to reduce the rate to an acceptable level. Offline, muons are required to pass quality requirements and to have $p_{\mathrm{T}}>5.5 \mathrm{GeV}$, to lie within the pseudorapidity range $|\eta|<2.4$, and to be isolated. Backgrounds arise from QCD processes, including non-prompt and fake muons, and from the residual tail of the $\Upsilon(1 \mathrm{~S})$ resonance in mass range 1.

Signal is extracted from a binned maximum likelihood fit to the dimuon mass spectrum. The QCD background is parameterised by a first-order polynomial, while the $\Upsilon(1 S), \Upsilon(2 S)$, and $\Upsilon(3 S)$ resonances are each paramterised by the sum of two Crystal Ball functions. The core resolution 

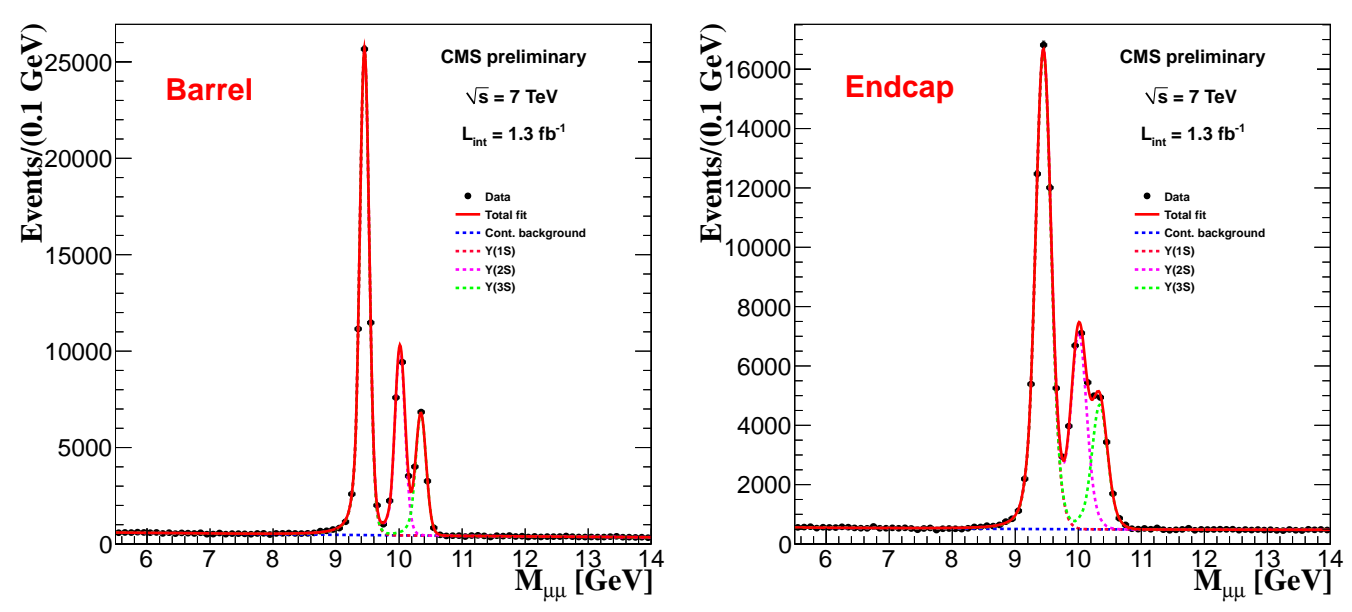

Figure 2: Background-only fits to the dimuon mass spectrum in the barrel (left) and endcap (right) data samples in the search for a light pseudoscalar $a$ in the context of the NMSSM. See text for details.

parameter is set by the $\Upsilon(1 \mathrm{~S})$, and constrained to scale with mass for the $2 \mathrm{~S}$ and $3 \mathrm{~S}$ resonsances. The mean of the $\Upsilon(1 S)$ peak is lef to vary in the fit in order to accomodate scale bias, while the masses of the $2 \mathrm{~S}$ and $3 \mathrm{~S}$ resonances are fixed by the known differences with the $1 \mathrm{~S}$ mass [15].

Figure 2 shows the results of background-only fits performed on the barrel and endcap data samples. For reference, expected NMSSM signals with assumed cross sections of $2 \mathrm{pb}$ (and scaled by a factor of 10) corresponding to masses of 7 and $12 \mathrm{GeV}$ are shown added to the total background contribution. To search for a signal, a scan is performed in ranges 1 and 2, divided into 110 and 100 steps of 30 mathrmMeV each, respectively. The signal is paramterised by a single Gaussian function with mean fixed to the center of the step and width determined by the expected mass resolution. Systematic uncertainties on the background contribution include uncertainty on the polynomial shape derived from testing alternative shapes, while a systematic uncertainty on the signal mass resolution is obtained from a comparison of the $\Upsilon(2 S)$ shape in data and simulation samples.

No significant signal is observed in either mass range. Figure 3 shows the resulting 95\% CL upper limits on the cross section times branching fraction for $\mathrm{pp} \rightarrow a \rightarrow \mu \mu$ derived from the mass scans. These limits can also be expressed as a function of the cosine of the mixing angle, which are also shown in Fig. 3 along with the corresponding limits from the BaBar experiment $[16,18]$. Limits are derived and shown for various values of $\tan \beta$ from 1 to 50 , and are superior in mass range 2 to previous limits [17, 19]. These results are published in Ref. [20].

\section{Search for a Doubly Charged Higgs Boson}

The minimal seesaw model of type II [21] includes one triplet scalar field that contains a doubly charged component $\Phi^{++}$. This particle decays to same-charge lepton pairs, including flavourviolating decays, and its Yukawa coupling matrix elements are proportional to the light neutrino 

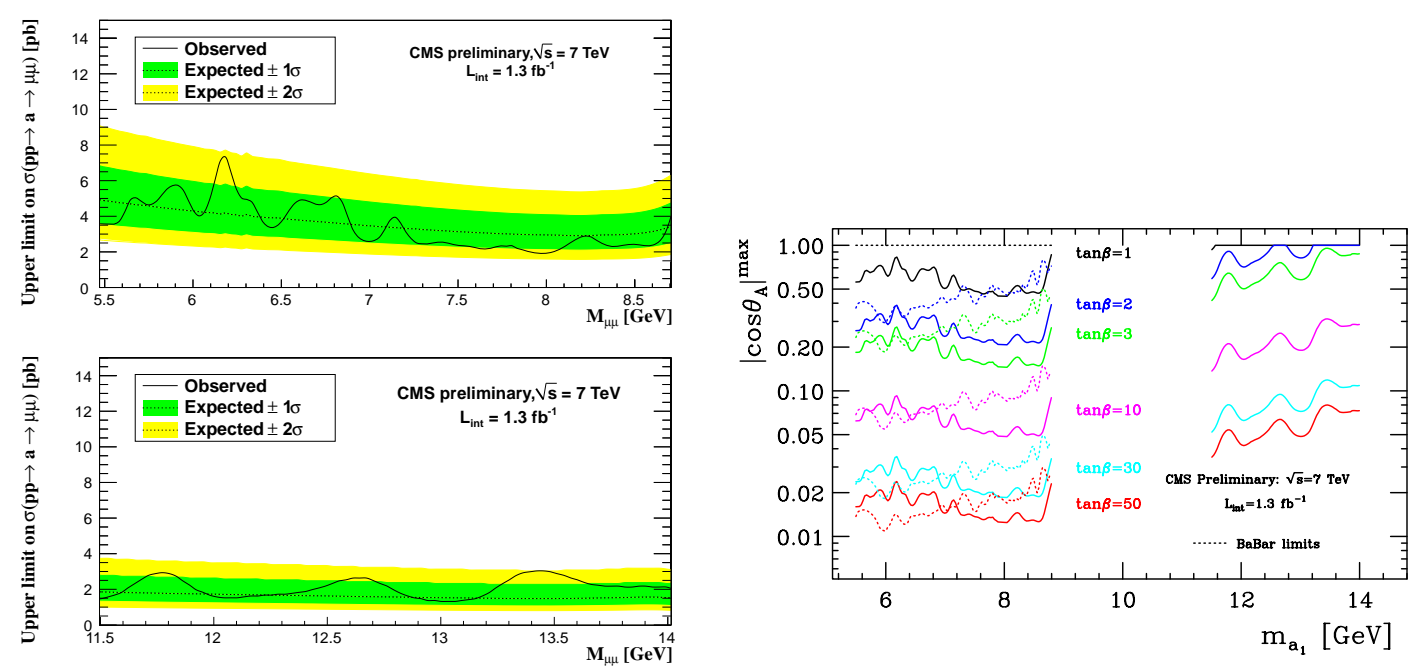

Figure 3: Results of the search for a light pseudoscalar Higgs boson decaying to muons. Left: 95\% CL upper limits on the cross section times branching fraction as a function of dimuon mass for mass range 1 (top) and mass range 2 (bottom). Right: Upper limits on the parameter $\left|\cos \theta_{A}\right|$ as a function of mass compared to similar limits from the BaBar experiment [16].

mass matrix. The observation of $\Phi^{++} \rightarrow \ell_{i}^{+} \ell_{j}^{+}$decays at the LHC would allow testing of the neutrino mass mechanism, including the absolute mass scale, hierarchy, and CP-violating phases.

The search presented here includes electron, muon, and tau final states in all flavour combinations, and uses a data sample of $4.6 \mathrm{fb}^{-1}$. Both the pair production $\left(\Phi^{++} \Phi^{--}\right)$and associated production $\left(\Phi^{++} \Phi^{-}\right)$channels are considered assuming degeneracy in their masses. The samples are separated in the three- and four-lepton final states corresponding to the different production mechanisms, and include one or up to two hadronically decaying tau leptons, respectively. In addition to a model-independent search, four benchmark points (BP1-BP4) are tested that probe different characteristics of the model [22].

The final state is distinguished by the presence of one or two resonant pairs of same-charge leptons, which is an extremely rare signature in the standard model. Muon candidates are required to satisfy $p_{\mathrm{T}}>5 \mathrm{GeV}$, whlie electrons hadronic taus are required to satisfy a threshold of $p_{\mathrm{T}}>15 \mathrm{GeV}$. Muon and electron candidates are required to be isolated to suppress backgrounds from misidentified tracks arising from QCD production of multijet events. Further background suppression is achieved from kinematic and topological information, including the scalar sum of the $p_{\mathrm{T}}$ of each lepton, and the opening angle between the same-charge leptons. The requirement of a fourth lepton reduces the background substantially. Figure 4 shows the distributions of three- and four-lepton masses in data compared to simulated backgrounds before the final selection is applied.

Signal is obtained by a sideband subtraction method using the background-enhanced regions before final selections are applied, where the background in the signal region is obtained from the background count in the sideband region and the ratio of signal-to-sideband yields obtained from simulation. For the $4 \tau$ search, where an explicit mass is not reconstructed, an "ABCD" method is employed, where the background yield in the signal region is extrapolated from three sideband regions defined by variables that have low correlations. Systematic uncertainties are dominated 

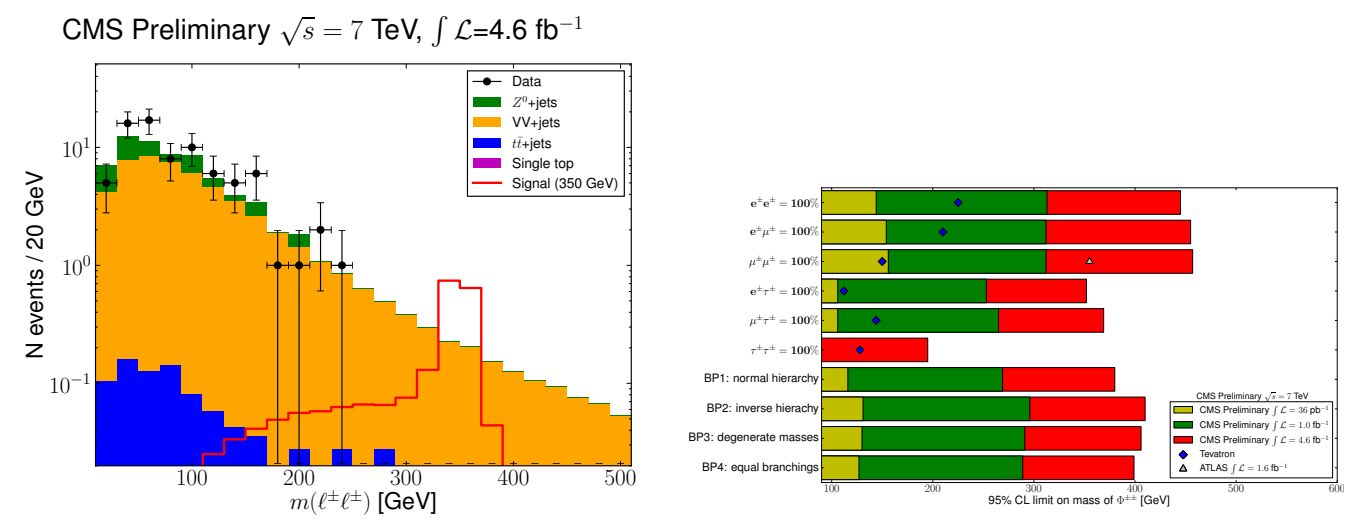

Figure 4: Left: distribution of dilepton mass from the four-lepton data sample before final selection. Right: 95\% CL lower limits on the $\Phi^{++}$mass from the different samples, and assuming different benchmark models.

by lepton ID and selection efficiencies, which are obtained from data wherever possible, and the statistical uncertainty of the background substraction.

No significant signals are observed in any final state. Figure 4 shows the resulting 95\% CL lower limits on the mass of the $\Phi^{++} / \Phi^{-}$for the inclusive search, and in the context of the specific benchmarks. Limits range from 200 to more than $400 \mathrm{GeV}$, and the results are summarized in more detail in Ref. [23].

\section{Summary}

Results of three separate searches for Higgs-like particles beyond the SM, and beyond the MSSM, were presented. No significant signals have been observed in any of the searches, yielding 95\% CL upper limits that rule out a SM4 Higgs boson with $m_{\mathrm{H}}>125 \mathrm{GeV}$, a light pseudoscalar Higgs boson in the mass region near the $\Upsilon$ resonances, and a doubly charged Higgs boson with masses above $200-400 \mathrm{GeV}$. These results use the $7 \mathrm{TeV}$ data only, and further searches using the $8, \mathrm{TeV}$ data set are underway and could yet reveal signs of physics beyond the SM.

\section{References}

[1] CMS Collaboration, "Observation of a new boson at a mass of $125 \mathrm{GeV}$ with the CMS experiment at the LHC," Phys. Lett. B716 (2012) 30-61.

[2] ATLAS Collaboration, "Observation of a new particle in the search for the Standard Model Higgs boson with the ATLAS detector at the LHC," Phys. Lett. B716 (2012) 1-29.

[3] CMS Collaboration, "The CMS experiment at the CERN LHC," JINST 03 (2008) S08004.

[4] G. D. Kribs, T. Plehn, M. Spannowsky, and T. M. P. Tait, "Four generations and Higgs physics," Phys. Rev. D 76 (2007) 075016.

[5] CDF and D0 Collaborations, "Colmbined Tevatron upper limit on $\mathrm{gg} \rightarrow \mathrm{H} \rightarrow \mathrm{W}^{+} \mathrm{W}^{-}$and constraints on the Higgs boson mass in fourth-generation fermion models," Phys. Rev. D 82 (2010) 011102. 
[6] CMS Collaboration, "Measurement of $\mathrm{W}^{+} \mathrm{W}^{-}$production and search for the Higgs boson in $\mathrm{pp}$ collisions at sqrts = 7 TeV," Phys. Lett. B699 (2011) 24-47.

[7] LHC Higgs Cross Section Working Group, S. Dittmaier, C. Mariotti, et al., "Handbook of LHC Higgs cross sections: 2. Differential distributions," CERN-2012-002 (CERN, Geneva, 2012), arXiv:1201.3084.

[8] CMS Collaboration, "Search for the standard model Higgs boson decaying into two photons in pp collisions at sqrts $=7 \mathrm{TeV}$, " Phys. Lett. B710 (2012) 403-425.

[9] CMS Collaboration, "Search for the standard model Higgs boson decaying to $\mathrm{W}^{+} \mathrm{W}^{-}$in the fully leptonic final state in pp collisions at sqrts = 7 TeV," Phys. Lett. B710 (2012) 91-113.

[10] CMS Collaboration, "Search for the standard model Higgs boson in the decay channel $\mathrm{H} \rightarrow \mathrm{ZZ} \rightarrow 4 \ell$ in pp collisions at sqrts $=7 \mathrm{TeV}$," Phys. Rev. Lett. 108 (2012) 111804; "Search for the standard model Higgs boson in the $\mathrm{H} \rightarrow \mathrm{ZZ} \rightarrow 2 \ell 2 v$ channel in pp collisions at sqrts $=7 \mathrm{TeV}, " \mathrm{JHEP} \mathbf{0 3}$ (2012) 040; "Search for the standard model Higgs boson in the decay channel $\mathrm{H} \rightarrow \mathrm{ZZ} \rightarrow q \bar{q} \ell^{-} \ell^{+}$in pp collisions at sqrts $=7 \mathrm{TeV}, "$ JHEP 04 (2012) 036.

[11] "Search for neutral Higgs bosons decaying to tau pairs in pp collisions at sqrts $=7 \mathrm{TeV}$," Phys. Lett. B713 (2012) 68-90.

[12] CMS Collaboration, "Combined results of searches for a Higgs boson in pp collisions at sqrts $=7 \mathrm{TeV}$ in the context of a standard model with four generations of fermions," CMS-HIG-12-013 (2012).

[13] M. Maniatis, Int. J. Mod. Phys. A 25 (2010) 3505.

[14] B. A. Dobrescu and K. T. Matchev, JHEP 09 (2000) 031.

[15] K. Nakamura et al. (Particle Data Group), J. Phys. G 37 (2010) 075021.

[16] B. Aubert et al. (BaBar Collaboration), Phys. Rev. Lett. 103 (2009) 081803; Phys. Rev. Lett. 103 (2009) 181801.

[17] G. Apollinari et al., Phys. Rev. D 72 (2005) 092003.

[18] R. Dermisek and J. F. Gunion, Phys. Rev. D 81 (2010) 075003.

[19] T. Aaltonen et al. (CDF Collaboration), Eur. Phys. J C 62 (2009) 319.

[20] CMS Collaboration, "Search for a light pseudoscalar Higgs boson in the dimuon decay channel in pp collisions at sqrts = 7 TeV," Phys. Rev. Lett. 109 (2012) 121801.

[21] M. Magg and C. Wetterich, "Neutrino mass problem and gauge hierarchy," Phys. Lett. B94 (1980) 61; J. Schechter and J. W. F. Valle, "Neutrino masses in SU(2) x U(1) theories," Phys. Rev. D22 (1980) 2227; G. Lazarides, Q. Shafi, and C. Wetterich, "Proton lifetime and fermion masses in an SO(10) model," Nucl. Phys. B181 (1981) 287; R. N. Mohapatra and G. Senjanovic, "Neutrino masses and mixings in gauge models with spontaneous parity violation," Phys. Rev. D23 (1981) 165.

[22] M. Kadastik, M. Raidal, and L. Rebane, "Direct determination of neutrino mass parameters at future colliders," Phys. Rev. D77 (2008) 115023.

[23] CMS Collaboration, "A search for a doubly-charged Higgs boson in pp collisions at $\sqrt{s} 7 \mathrm{TeV}$," arXiv:1207.2666 (2012). 